\title{
First-Principles Study of Dissociation Processes for the Synthesis of Fe and Co Oxide Nanoparticles
}

\author{
Burak Özdamar, ${ }^{\ddagger}$ Assil Bouzid, ${ }^{\text {I[C }}$ Guido Ori, Carlo Massobrio, and Mauro Boero*(e) \\ University of Strasbourg, Institut de Physique et Chimie des Matériaux de Strasbourg (IPCMS), CNRS UMR 7504, 23 rue du Loess, \\ F-67034 Strasbourg, France
}

\section{Supporting Information}

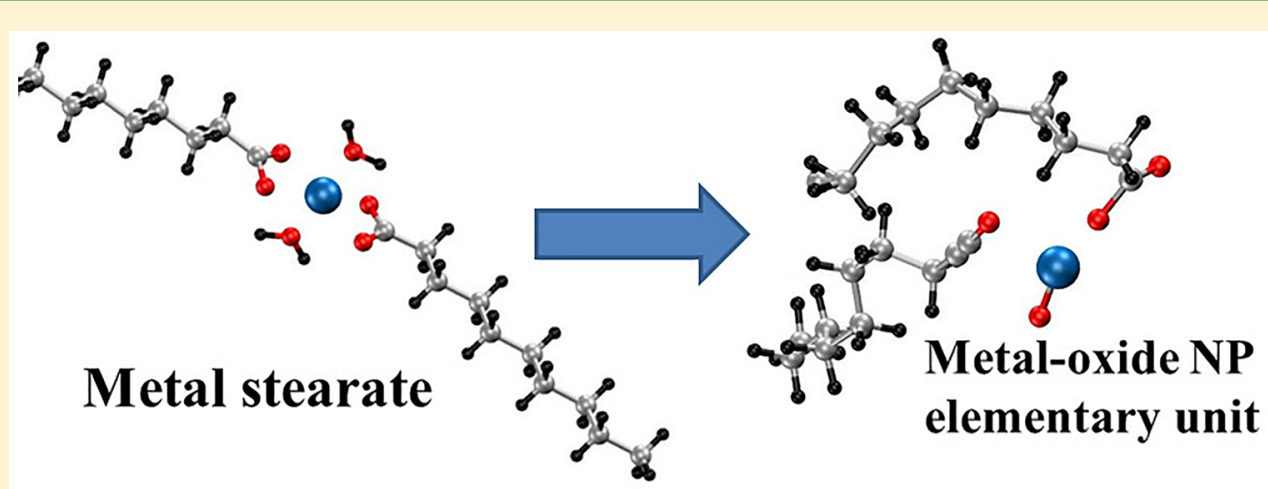

ABSTRACT: Thermal decomposition is a practical and reliable tool to synthesize nanoparticles with monodisperse size distribution and reproducible accuracy. The nature of the precursor molecules and their interaction with the environment during the synthesis process have a direct impact on the resulting nanoparticles. Our study focuses on widely used transition-metal (Co, $\mathrm{Fe}$ ) stearates precursors and their thermal decomposition reaction pathway. We show how the nature of the metal and the presence or absence of water molecules, directly related to the humidity conditions during the synthesis process, affect the decomposition mechanism and the resulting transition-metal oxide building blocks. This, in turn, has a direct effect on the physical and chemical properties of the produced nanoparticles and deeply influences their composition and morphology.

\section{INTRODUCTION}

Functionalized metal and metal oxide nanoparticles (NPs) represent forefront research worldwide because of their broad range of applications from magnetic and electronic devices to biomedical applications. Specifically, and yet not exhaustively, applications are targeting data storage units and magnetic fluids for micro- and nanoelectromechanical system (MEMS/NEMS) devices, image contrasting agents for magnetic resonance imaging, and drug delivery modules. ${ }^{1-7}$ Nonetheless, the production of NPs with desired size, morphology, and functionalities starts from a careful choice of the precursors and the processes adopted to extract the building blocks for their synthesis. ${ }^{8-10}$ Among the most popular precursors, stearates play a major role. ${ }^{11}$ The fatty acid compounds consist generally of a metallic center surrounded by organic ligands. Interactions with the ligands and the presence of detectable impurities contaminating the precursors play non-negligible roles in affecting the size distribution and morphology of NPs. ${ }^{12}$ Water, often, acts as a functional impurity that can affect the precursor conversion rates or hydrolyze the NPs surface. Other sources causing water contamination during NPs growth are also possible, such as the ketonization of unreacted acids under growth conditions. Even small amounts of water impurities $(0.02-0.1 \mathrm{~mol} / \mathrm{mol}$ water:precursor) have been shown to impact negatively the NPs growth by limiting its size tunability. ${ }^{13}$ Indeed, the precursor chemistry and the rate of precursor conversion are important factors controlling NPs formation and growth, with possible unexpected influences on the NPs size and size distribution. ${ }^{14,15}$ This makes the control of the NPs precursors a delicate issue, especially for small NPs below $20 \mathrm{~nm}$, which are the major target for the applications mentioned above. ${ }^{16-18}$ This, in turn, calls for special attention to the role of the ligand-NPs interactions starting from the NPs seed formation to the nucleation-to-growth process and to the postfunctionalization stages. All these subsequent steps are responsible for the final properties of the nano-object.

Current stearates-based synthesis methods used to produce NPs represent to date a rather controlled and versatile way to obtain NPs with the desired size distributions, tunable sizes, and, to a certain extent, specific magnetic and electronic properties, yet the initial formation of the elementary building blocks for NPs is still escaping fine control since they crucially depend on the experimental conditions and the environment in which the precursors are decomposed. The spontaneous thermal decomposition ${ }^{19}$ is one of the most effective methods

Received: August 15, 2017

Published: December 5, 2017 
(a)

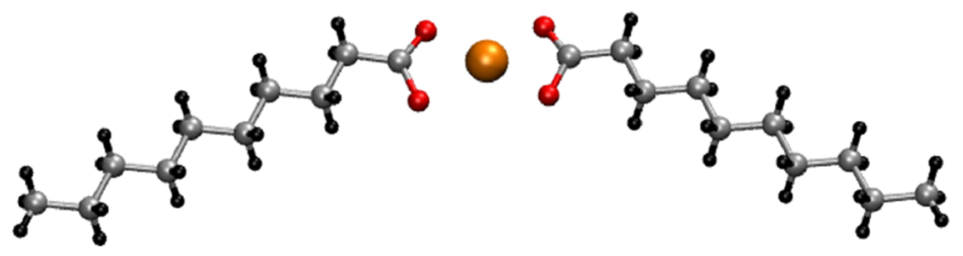

(b)

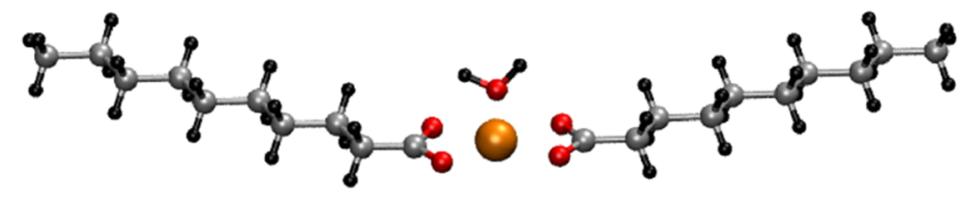

(c)

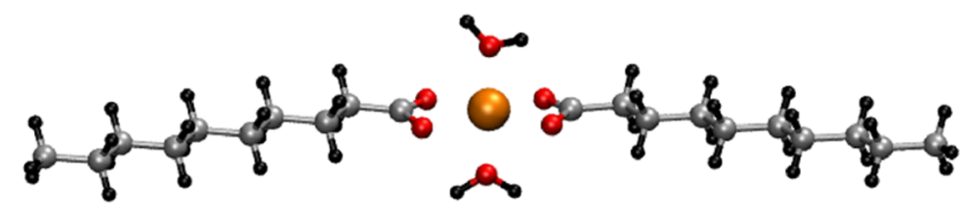

Figure 1. Initial stable configurations of the TM-stearate compounds, $\mathrm{TM}=\mathrm{Fe}$, Co. (a) In dry conditions, (b) with one $\mathrm{H}_{2} \mathrm{O}$ molecule, i.e. octahedral TM-O coordination with one vacant site, and (c) with two $\mathrm{H}_{2} \mathrm{O}$ molecules, i.e. octahedral TM-O coordination. The color code is black for $\mathrm{H}$, gray for $\mathrm{C}$, red for $\mathrm{O}$, and orange for the TM.

to fine control the size distribution and morphology of nearly monodisperse metal oxide NPs with significantly high yield. Such a versatile aspect is the result of the fast reaction that thermal decomposition triggers in stearates. ${ }^{20,21}$ The enhanced temperature triggers the release of the long hydrocarbon chains containing a carboxyl group present in the stearate precursor and surrounding the metal center. Such a decomposition generally occurs in a nonaqueous solvent and allows for control of the size and morphology of the resulting NPs, as well as their in situ functionalization, thereby limiting their aggregation in suspension.

Metallic complexes carrying as ligands fatty acid salts such as acetates, myristates, oleates, and stearates ${ }^{21}$ used as NPs precursors share as a common feature the characteristic coordination of two or more organic ligands in a bidentate arrangement around a transition metal (TM). ${ }^{22}$ NPs are formed through the thermal decomposition of these complexes, consisting of the release of the organic ligands, and subsequent aggregation of the unscreened metal atoms. However, to date, little attention has been given to these molecular precursors, and most of the synthesis procedures rely more on empirical trial and error attempts rather than on a systematic atomic-level insight into the nature of these stearates and the microscopic mechanisms regulating their decomposition. An open question is the evolution of the metal coordination during the process and, specifically, whether or not the bidentate configuration is kept or if a monodentate one can be realized. Moreover, interactions of impurities with these precursors are a major source of problems in terms of stability and nature of the elementary building block produced which, in turn, will contribute to the formation of the NP. ${ }^{23}$ Specifically, the sizes of the NPs tend to diminish if water molecules are present during the synthesis reaction. ${ }^{17,24}$ Both $\mathrm{Fe}$ and Co stearate complexes are in a 4-fold and planar coordination in the pristine molecular geometry, ${ }^{25,26}$ with the oxygens of the carboxylate moiety $\left(\mathrm{RCOO}^{-}\right)$forming a plane. If water molecules are present, then they could approach the metal site and coordinate around it, presumably along the directions roughly orthogonal to the $\mathrm{RCOO}^{-}$plane to complete the typical hydration shell of cations. ${ }^{27-29}$ Depending on the number of $\mathrm{H}_{2} \mathrm{O}$ molecules, a 5- (approach of one water molecule) or 6-fold coordination (approach of two $\mathrm{H}_{2} \mathrm{O}$ molecules from the two opposite sides) can be realized. These additional water molecules have a direct effect on both the thermal decomposition mechanism and the related activation energies. As a consequence, unpredictable effects arise in the synthesis of NPs, from an experimentally observed slowing down of the nucleation and growth to a complete hindering of the NPs formation. ${ }^{24}$

The complexity of the thermal decomposition process, the practical difficulty in isolating the early stage NPs seeds, and the general lack of experimental information about the nature of NPs building blocks motivate the use of first-principles simulations. The method is ideally suited to shed light on the short-lived precursors and complexes in the early stage and growth of NPs. ${ }^{30,31}$ The scope of our work is to provide answers to both the issue of the bidentate or monodentate coordination and to shed some light into the reaction pathway realized by stearates under dry and hydrated conditions. To this aim, we focus on single metal-stearate molecules in dry and microsolvation conditions to address a precise quest prompted by elusive experimental findings to be rationalized. ${ }^{17,24,32}$ In fact, the major difficulty is to disentangle and probe the effects of water on the release of the metal center. Moreover, equally unclear is whether and how water contributes to the formation of the nanoparticle seed. In the lack of any former simulation on these systems, unraveling these details calls for special attention to the local chemical nature of the metal and the $\mathrm{O}$ atoms directly coordinated to it. The surrounding solvent does not approach the hydrophobic hydrocarbon chains and is not directly involved in the formation of the metal oxide primitive building block. In our study, we make use of first-principles molecular dynamics approaches, enhanced by free energy sampling techniques for the simulation of the decomposition 
processes. This study provides also an insight into the effect of the different nature of the metal center, iron and cobalt, on the decomposition processes responsible for the formation of the elementary building block (Metal? Metal oxide? Or?) concurring to form the NP.

\section{METHODS}

In our simulations, both iron and cobalt stearate molecules have been considered. The ligands adopted in our simulations $\left(\mathrm{CH}_{3}-\left(\mathrm{CH}_{2}\right)_{8}-\mathrm{COO}\right)$ refer to laboratory stearates and are considerably shorter than the analogous commercial products (e.g., $\mathrm{CH}_{3}-\left(\mathrm{CH}_{2}\right)_{16}-\mathrm{COO}$ for the smallest compound, CAS number 5136-76-5). These have then a reduced flexibility and allow for avoiding complex folding processes that could screen the metal center. Besides dry conditions, we inspected also the possible degrees of hydration as sketched in Figure 1.

We make use of first-principles molecular dynamics (FPMD) simulations $^{33}$ within the density functional theory (DFT) ${ }^{34}$ framework as implemented in the $\mathrm{CPMD}^{35}$ code. The Becke exchange and the Lee-Yang-Parr correlation functional (BLYP) have been used to describe the exchange and correlation contributions. ${ }^{36,37}$ Core-valence interactions have been described by norm-conserving Troullier-Martins pseudopotentials (PPs) ${ }^{38}$ for $\mathrm{O}, \mathrm{C}$, and $\mathrm{H}$, while for transition metals $(\mathrm{Fe}, \mathrm{Co})$ we resorted to Goedecker-Teter-Hutter ${ }^{39-41}$ semicore PPs. Valence electron orbitals have been represented in a plane wave (PW) basis set with a cutoff energy of $80 \mathrm{Ry}$. To eliminate the problem of periodically repeated images, typical of standard PW approaches, an isolated cell ${ }^{42}$ with an edge of $33.87 \AA$ was used. Since experiment makes use of relatively diluted stearates, ${ }^{32}$ the release of periodic boundary conditions ensures that interactions with a neighbor ligand is absent all along the simulations. A spin-unrestricted approach is adopted in all the simulations, and van der Waals interactions were included according to Grimme's D2 formula. ${ }^{43}$ Free energy profiles for the various desorption processes have been sampled according to the Blue Moon ensemble (BME) approach. ${ }^{44,45}$ To this aim, different reaction coordinates were considered, as specified in the following section for each case. Generally, these reaction coordinates account for the distance TM-COO between the metal center and the carboxylate group of the coordinated ligand. They are increased gradually by increments of about $0.10 \AA$ to follow the dissociation process. The minimum equilibration time for each TM-COO constrained value was $\sim 3$ ps to ensure that both the average constraint force and the total energy of the system converge to an ergodic limit as the simulation evolves in time. As a check of the performance of our functional choice, we did one additional simulation for the case of the hydrated Co stearate with the hybrid PBE0 functional. $^{46}$ These results are given in the Supporting Information and basically confirm the analysis of the group of Grimme $^{47}$ and, specifically for Fe and Co, our own benchmark on metal-organic systems. ${ }^{48}$ Namely, our computational setup allows for one of the best compromises between accuracy and computational efficiency. We are reminded that although statistical errors in the free energy sampling can be reduced by increasing the statistics, accuracies are bound by the underlying DFT level used, hence an error bar of 1-2 kcal/ $\mathrm{mol}^{49}$ affects all values reported in the ongoing discussion. A fictitious electronic mass of 340.0 au and an integration step of $4.0 \mathrm{au}\left(9.675 \times 10^{-5} \mathrm{ps}\right)$ ensured good numerical control of the constants of motion.

\section{RESULTS AND DISCUSSION}

TM-Stearates in Dry Conditions. Starting from the Febased stearate system in dry conditions, we first optimized the molecular structure via a damped molecular dynamics run. ${ }^{50}$ Subsequently, we equilibrated the geometry at room temperature $(300 \mathrm{~K})$ in a canonical NVT ensemble, ${ }^{51-54}$ obtaining the stable configuration shown in panel (a) of Figure 1. The equilibrium $\mathrm{Fe}-\mathrm{O}$ coordination distance turns out to be $2.00 \AA$, a value rather standard in the coordination chemistry of ironoxygen compounds. ${ }^{22,55}$ On the other hand, the high flexibility of the hydrocarbon chain displays some structural fluctuations and nonplanar ligand geometries at finite temperature. This is not unexpected and does not alter the coordination of the metal center. Since an activation barrier larger than $k_{\mathrm{B}} T(T=$ temperature, $k_{\mathrm{B}}=$ Boltzmann's constant) exists to remove the ligand from the metal center, the decomposition could not be observed on the ps time scale of standard FPMD simulations. Thus, we resorted to BME simulations to work out free energy barriers and to sample the reaction pathway leading to the expected dissociation. In our first simulation, the chosen reaction coordinate was the distance between the Fe atom and the $-\mathrm{COO}$ moiety of the ligand (measured as the $\mathrm{Fe}-\mathrm{C}$ distance) whose equilibrium value is $d_{\mathrm{Fe}-\mathrm{COO}}=2.31 \AA$. Subsequent increasing of this distance, by increments of 0.1 $\AA$, leads eventually to the detachment of the coordinated ligand from the metal center. The free and total energy profiles for this dissociation are shown in Figure 2, and the main steps of the

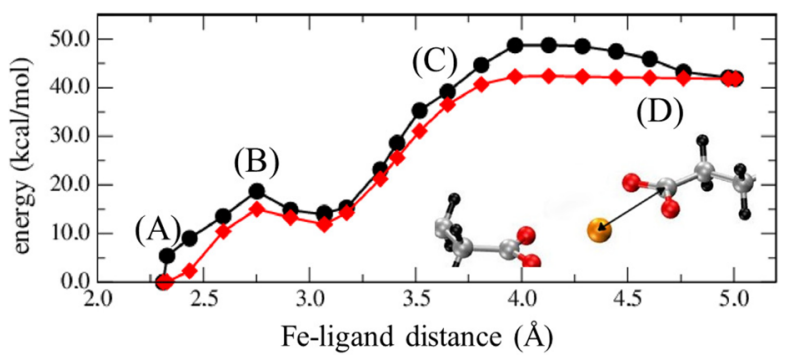

Figure 2. Free (red) and total (black) energy profiles for the desorption of one of the two ligands coordinated to the metallic Fe center in Fe-based stearates in dry conditions. The arrow in the inset shows the selected reaction coordinate metal-COO.

process along the depicted reaction pathway are sketched in Figure 3, where panels (A), (B), (C), and (D) correspond to values of the reaction coordinate of $2.31,3.07,3.52$, and $4.52 \AA$, respectively. We recall that the free energy profile is given by the BME thermodynamic integration, whereas the total energy is directly computed as the sum of the Kohn-Sham DFT energy plus the ionic kinetic energy, i.e. $E_{\text {tot }}=E^{\mathrm{DFT}}+(1 /$ 2) $\sum_{I} M_{I} \dot{\mathbf{R}}_{I}^{2}$ with $M_{I}$ and $\dot{\mathbf{R}}_{I}$ being the mass and the velocity of the ion $I$, respectively. The monotonic increase in both the total (black curve) and free energy (red curve) shown in Figure 2 continues until $d_{\mathrm{Fe}-\mathrm{COO}}$ reaches $2.75 \AA$. Here, the bidentate coordination of the ligand to the metal center becomes monodentate as shown in panel (B) of Figure 3, and the square planar coordination of $\mathrm{Fe}$ begins to be compromised.

Being less coordinated to $\mathrm{Fe}$, the detaching chain becomes even more flexible. This entropic contribution is visible in the total energy profile which is generally larger than the free energy curve $\left(\Delta E_{\text {tot }}=\Delta F+T \Delta S\right)$. We remark that despite the intrinsic flexibility of the ligands, these are not long enough to give rise to folding processes able to screen completely the 


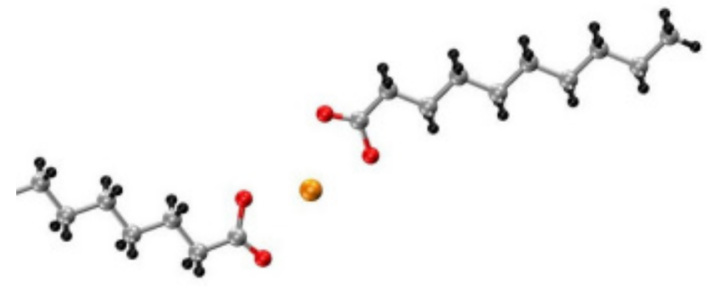

(A)

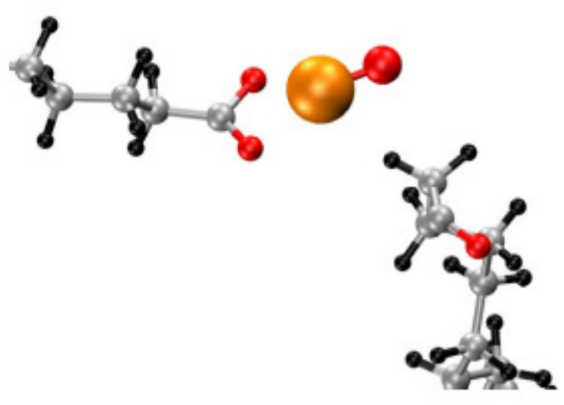

(C)

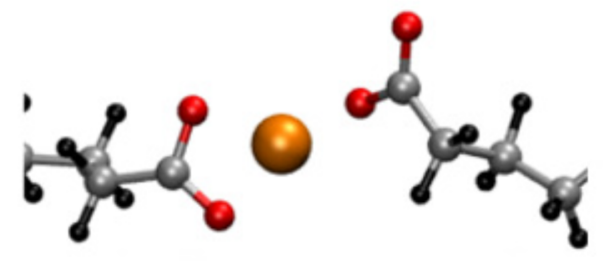

(B)

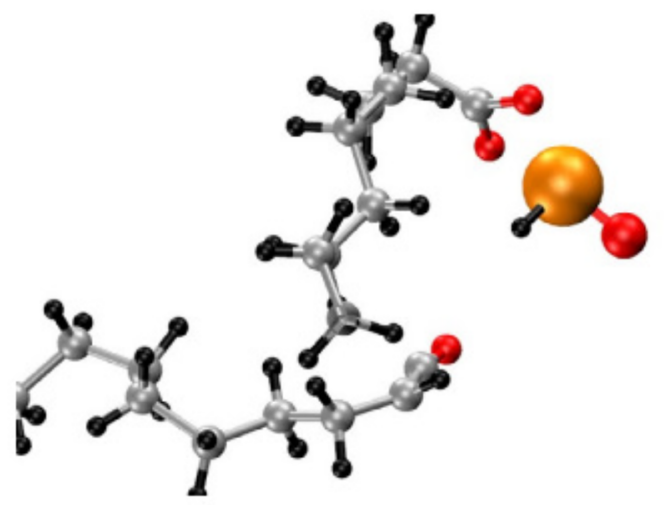

(D)

Figure 3. Structural evolution of the iron stearate in dry conditions during the constrained dynamics. The ligand on the left in panel (A) is the one that is gradually displaced away from the metallic Fe center. A first process occurring is the transition from a bidentate to a monodentate (B) coordination. Eventually, an oxidized iron is produced (C), and the first $\mathrm{FeO}$ seed is formed. Occasionally a temporary hydrogen transfer can occur (D). Labeling follows the one given in Figure 2. The color code for atoms is identical to Figure 1.

metal center. In fact, the entropy contribution is rather limited as the difference between the total and free energy profiles shows. Additional details are provided in Table S1 of the Supporting Information. After overcoming a transition state characterized by the first maximum $\Delta F=15.02 \mathrm{kcal} / \mathrm{mol}(0.65$ $\mathrm{eV}$ ) at a value of $2.75 \AA$ of the reaction coordinate, the bidentate configuration becomes monodentate. One of the two $\mathrm{O}$ atoms of the ligand is still shared with the metal center until a distance $d_{\mathrm{Fe}-\mathrm{COO}}=3.52 \AA$ is reached. This corresponds to the situation sketched in panel (C) of Figure 3. From this point on, the ligand is no longer bound to the Fe center, and one of the two $\mathrm{O}$ atoms of the - $\mathrm{COO}$ group departs from the ligand and forms a stable bond with the metal center: This is the first $\mathrm{Fe}-$ $\mathrm{O}$ seed in the NP growth mechanism. By continuing the simulation, we observed that occasionally a hydrogen transfer from the ligand to the metal center can occur, ${ }^{56-58}$ as shown in panel (D) of the same figure. This is however not a stable condition, and the $\mathrm{H}$ atom on the Fe center is spontaneously transferred back to the chain. These results are consistent with the experimental finding of regular iron oxide NPs synthesized from these stearates. ${ }^{18}$ Because of our choice of reaction coordinate, the long plateau of the free energy (Figure 2) starting from $d_{\mathrm{Fe}-\mathrm{COO}} \sim 4.00 \AA$ indicates that the interaction of the iron with the hydrocarbon chain is terminated. An auxiliary BME simulation performed by using as a reaction coordinate the center of mass of the two oxygen atoms in the $-\mathrm{COO}$ group, instead of the central $\mathrm{C}$ atoms, has given an analogous result in terms of both free energy profile and dissociation mechanism.

Co-based stearates have been studied following the same protocol and simulation conditions used for the Fe-based ones. Also in this case, in an attempt at checking whether or not the choice of the reaction coordinate can influence the reaction pathway, two independent BME simulations with two different reaction coordinates were done. As in the Fe-based systems, we used either the Co-COO distance $\left(d_{1}\right.$ in the upper panel of Figure 4) or the distance between the metal center and the center of mass of the two oxygen atoms belonging to the -COO group ( $d_{2}$ in the lower panel of Figure 4$)$.

Although the overall mechanism depicted by both BME simulations is identical in terms of dissociation and final product, the free energy profiles are slightly different. Clearly, in the initial stage the equilibrium distances read $d_{1}=2.31$ and $d_{2}$ $=1.82 \AA$, respectively. As the simulation proceeds, the free energy barrier separating the bidentate from the monodentate configuration become $20.85 \mathrm{kcal} / \mathrm{mol}(0.90 \mathrm{eV})$ when $d_{1}=2.75$ $\AA$ in the first case, whereas it reduces to $12.22 \mathrm{kcal} / \mathrm{mol}(0.53$ $\mathrm{eV}$ ) when $d_{2}=2.17 \AA$. For comparison, the realization of a monodentate structure in the case of the Fe-based stearate occurred for an identical value of the reaction coordinate $d_{1}$ but with a lower $(15.02 \mathrm{kcal} / \mathrm{mol})$ free energy barrier. This is a clear indication of the fact that the dissociation of iron stearates is energetically less demanding than the dissociation of analogous Co-based compounds in identical thermodynamic conditions in the absence of impurities. Also in this case, the rate limiting step is the release of an $\mathrm{O}$ atom from the firstcoordination shell of Co to form the initial metal oxide elementary building block of the NP formation. Unfortunately, this is hard to realize in the case of Co-based stearates. In fact, after an initial trend not too different from the one of the $\mathrm{Fe}$ based system, BME simulations show a monotonic increase in the free energy profile with a final product being a hydrated Co atom as shown in Figure 5. 

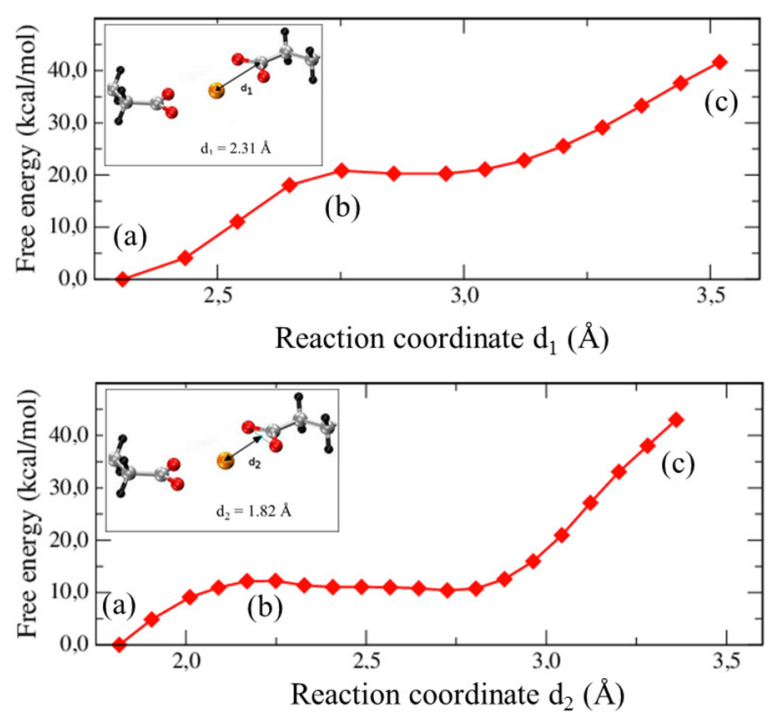

Figure 4. Free energy profiles obtained with the two different reaction coordinates. The initial equilibrium distances differ because of their definitions: In the upper panel the distance of the metal atom from the carbon atom of the carboxylate group is used $\left(d_{1}=2.31 \AA\right.$ ), whereas the separation between the Co atom and the center of mass of the oxygen atoms of the carboxylate group $\left(d_{2}=1.82 \AA\right)$ is used in the lower panel.

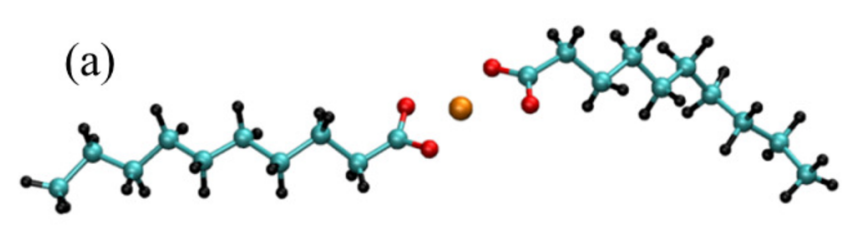

(b)

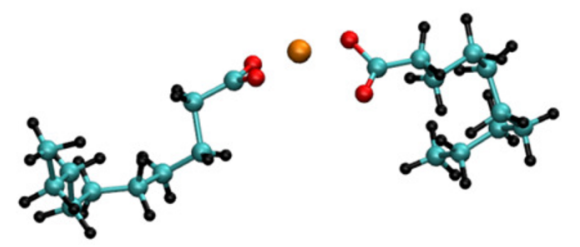

(c)

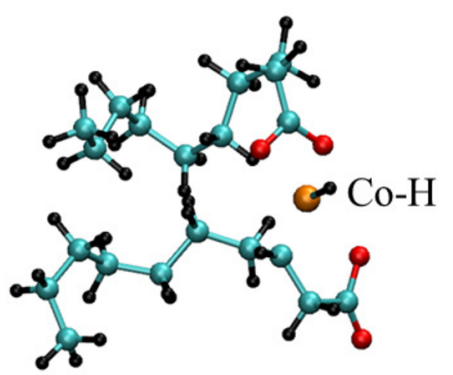

Figure 5. Main stages of the dissociation mechanism of Co-based stearates. The labeling beside each panel refers to the points with identical notations along the free energy profiles of Figure 4.

This fundamental finding can be inferred to be one of the main reasons at the origin of the observed discrepancy in the formation of iron and cobalt NPs via thermal decomposition. Despite identical experimental conditions, the synthesis of Co oxide nanoparticles poses severe limitations, resulting in a rather uncontrolled spherical morphology different from what can be achieved with $\mathrm{Fe}_{3} \mathrm{O}_{4} \mathrm{NPs}^{24}$ The experimental evidence to the fact that the cobalt stearate decomposition requires higher temperature ${ }^{24}$ in comparison with its iron counterpart is legitimized by the higher energy barrier found by our simulations.

TM-Stearates in Partial Hydration Conditions. Within an identical simulation protocol, one water molecule approached the metal center of the stearate. This additional $\mathrm{H}_{2} \mathrm{O}$ molecule is initially coordinated to the metal and, with the $\mathrm{O}$ atom pointing to the metallic cations, belongs to the coordination shell of either $\mathrm{Fe}$ or Co. The initial configuration is what is shown in Figure 1(b), yet upon dynamics, either unconstrained or within a BME simulation framework, the $\mathrm{H}_{2} \mathrm{O}$ molecule in the (defective) octahedral hydration shell site around $\mathrm{Fe}$ oscillates, initially keeping an average distance of 3.7 $\AA$ from the metal center but then escaping from the $\mathrm{Fe}$ coordination shell, as shown in panel (a) of Figure 6. The escape of the water molecule leaves the stearate in a condition identical to the dry case, and, for this reason, the free energy profile does not differ from what has been found for a dry $\mathrm{Fe}-$ stearate for the departure of one of the two ligands. Indeed, the $\mathrm{BME}$ simulation proceeds along the same reaction mechanism depicted in the former paragraph. The only point worthy of note is the fact that the water molecule switches its position, instantaneously and temporarily, within the coordination shell of iron from a vertex of an octahedron to a planar structure like the one shown in Figure 6(b).

In these conditions, the $\mathrm{H}_{2} \mathrm{O}$ molecule is inserted between the $-\mathrm{COO}$ moiety of the ligand and the metal center. As a consequence, the stearate ligand which was initially in a bidentate chelate configuration switches to a bridging-like configuration with one oxygen atom of the $-\mathrm{COO}$ group pointing to one of the protons of the $\mathrm{H}_{2} \mathrm{O}$ and the other oxygen atom pointing to the metal center. Actually, the proton of the water molecule pointing at one of the $\mathrm{O}$ sites of the $-\mathrm{COO}$ group becomes a shared proton as in a Zundel-like complex. ${ }^{59-61}$ This specific $\mathrm{H}^{+}$jumps continuously from the $\mathrm{H}_{2} \mathrm{O}$ molecule to the $\mathrm{O}$ atom of the ligand, as expected in Zundel complexes. When this occurs during a BME simulation, we observed that the first free energy barrier for the dissociation, corresponding to the maximum located at 2.75 $\AA ̊$ in Figure 2, increases by $\sim 25$ compared to its dry analogue. This is consistent with the fact that the Fe stearate with the inserted water molecule is lower in terms of total energy by about $6 \mathrm{kcal} / \mathrm{mol}$ with respect to the defective octahedral arrangement. This structural change in the number of $\mathrm{O}$ atoms coordinated to the $\mathrm{Fe}$ center is reflected in a change of oxidation state of iron. In fact, calculation of the Bader charge ${ }^{62}$ done on the two different arrangements has shown a net charge on $\mathrm{Fe}$ of +2.6 for the defective octahedron, hence closer to $\mathrm{Fe}(\mathrm{III})$, while for the planar geometry with the water molecule bridging the metal center and the ligand we got +1.7 , hence closer to $\mathrm{Fe}(\mathrm{II})$. However, as a consequence of the water molecule desorption, such a partially hydrated system evolves as in dry conditions, and a $\mathrm{FeO}$ seed is formed with a mechanism identical to the one discussed in the former paragraph. Although the overall barrier of the rate limiting step remains unchanged, as well as the reaction mechanism, this indicates that the desorption of this water molecule, which eventually occurs, requires an additional amount of energy. Ongoing experiments ${ }^{17,24}$ make use of $\mathrm{Fe}$ - and Co-based stearates thermally decomposed in a high boiling point organic solvent, yet results in terms of size, shape, and properties of the NPs depend in an unclear way on the humidity conditions and are affected by unsolved difficulties in drying stearates, 


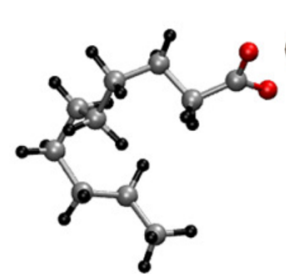

(a)

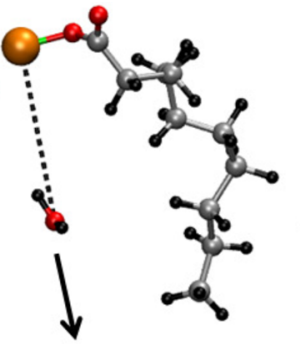

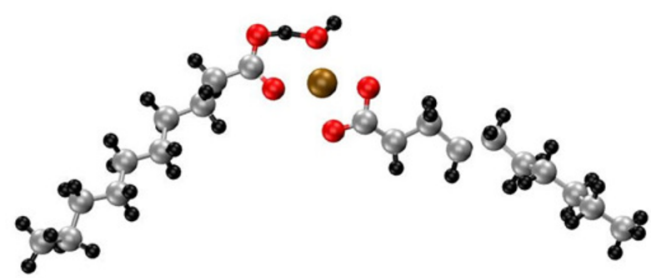

(b)

Figure 6. Escape of a single water molecule from the hydration shell of the metal center (a) and insertion of the water molecule between the Fe center and the $-\mathrm{COO}$ group of the ligand (b). The color code is identical to that of Figure 1.

(a)

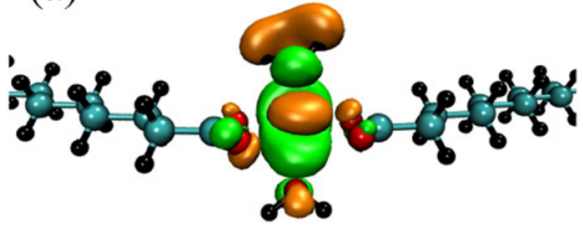

(b)

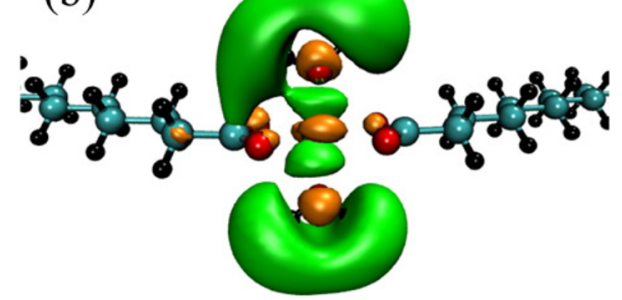

Figure 7. HOMO (a) and LUMO (b) orbitals of the Fe-based stearate in the presence of two water molecules at the initial equilibrium distance $d_{\mathrm{Fe}-\mathrm{COO}}=2.43 \AA$. Isosurfaces are shown at values of $\pm 0.025\left(\mathrm{e} / \AA^{3}\right)^{1 / 2}$ in orange (positive values) and green (negative values). The color code is identical to that of Figure 1 apart from $\mathrm{C}$ atoms here in cyan color for clarity.

especially Fe-based stearates, previously exposed to aqueous environments. Our simulations address explicitly this point. We do not make any claim about the experimental percentage of water impurities; yet being that the ligand is fully hydrophobic, $\mathrm{H}_{2} \mathrm{O}$ molecules can be accommodated only in the vicinity of the metal center, and because of the double -COO coordination, only one or two water molecules can fill the coordination shell of the metal. This is the focus of the ongoing discussion and a standard approach in the study of the role of specific water molecules in transition-metal compounds carrying ligands. ${ }^{63}$ Moreover, equally unclear is whether and how water contributes to the formation of the NP seed. Our results confirm the hypothesis that whenever water molecules are not immediately released, they become part of the complex. As such, it becomes energetically more demanding to remove $\mathrm{H}_{2} \mathrm{O}$ from a hydrated stearate having assumed a bridging conformation.

The simulation of the cobalt-based system in the presence of one water molecule, initially accommodated in the hydration shell of the metal center, was performed with the identical protocol used for the Fe-based system. However, both unconstrained and BME simulations have shown that the $\mathrm{H}_{2} \mathrm{O}$ molecule leaves the coordination shell of the Co atom. The system then reverts quickly to dry conditions, and both dissociation mechanism and free energy barrier do not differ from what has been discussed in the former paragraph. To verify this behavior, simulations have been repeated by imposing an additional constraint on the water-cobalt distance during the NVT equilibration in order to stabilize the water molecule. Nonetheless, as soon as this second constraint is released, nothing prevents the escape of the $\mathrm{H}_{2} \mathrm{O}$ molecule away from the metal center. We can then infer that hydrating the Co-based stearate is more difficult than in the case of their Fe-based analogous compounds. As a general comment, we remark that in transition metals, the kinetics of the water exchange mechanism strongly depends on the number of occupied $d$-electrons and therefore the ionic radius. ${ }^{64}$ The presence of one additional diffuse d-orbital in the electronic configuration of Co $\left([\mathrm{Ar}] 3 d^{7} 4 s^{2}\right)$, as opposed to the case of Fe ( $\left.[\mathrm{Ar}] 3 d^{6} 4 s^{2}\right)$, seems to be sufficient to trigger such a different behavior. As a result, an immediate release of the water molecule occurs instead of its insertion between the metal center and the ligand. Furthermore, since the interaction of the water and the metal center is a short lasting event, the free energy barrier and the transition state are not affected by its presence.

The general scenario that can be inferred is that the presence of one water molecule causes an increase in the energy barrier for the Fe-based system by as much as $25 \%$, whereas it does not affect the Co-based system because of the short-living waterstearate complex which leaves the system always in dry conditions for the dissociation process. The resulting nanoparticle seed, in any case, remains unaffected apart from differences in the free energy profiles in the case of Fe-based stearates. On the basis of these results, it can be concluded that $\mathrm{Fe}$ and Co present some difficulties in accommodating a single water molecule in what would result as an incomplete octahedral geometry around the metal center. This is due to the preferred coordination geometry (and number) of $\mathrm{O}$ atoms around either $\mathrm{Fe}$ or $\mathrm{Co}$ in $\mathrm{a}+2$ oxidation state, namely either tetrahedral (4-fold) or octahedral (6-fold). ${ }^{27,28,65}$

TM-Stearates in Full Hydration Conditions. The maximum degree of hydration of Fe- and Co-based stearates is the one shown in Figure 1(c). In these conditions, water molecules are located orthogonal to the plane made by the four $\mathrm{O}$ atoms of the stearate ligand, thus completing the 6-fold hydration shell of the metal cations. On experimental grounds, ${ }^{18,24}$ it was suggested that the ligand desorption process would follow a different pathway as a consequence of the presence of water in proximity of the metal center, probably as a result of hydrogen bonds formed between the $\mathrm{H}_{2} \mathrm{O}$ molecules and the carboxylate groups of the ligand. This was 
actually inferred a posteriori by inspecting the morphology and structure of the Co- and Fe-based NPs obtained in different humidity conditions. Here we focus on this issue to complement experiments and to provide insight into the microscopic origin of the different dissociation process hypothesized.

Upon equilibration, the electronic structure around the metal center of the hydrated Fe-based stearate in terms of higher occupied (HOMO) and lowest occupied (LUMO) orbitals is the one reported in Figure 7.

The energy difference between the HOMO and the LUMO states is $1.23 \mathrm{eV}$, a value that did not undergo any change during the dissociation process; yet the shape of these orbitals is already instructive. The HOMO states of the system is a $d_{x y}$ orbital from the metal center with additional lobes coming mainly from the $p$ states of the oxygen atoms belonging to the $-\mathrm{COO}^{-}$group plus contributions from the water molecules. More precisely, one can recognize in the HOMO the lone pairs of the $\mathrm{O}$ atoms of the $\mathrm{H}_{2} \mathrm{O}$ molecules plus a small amount of the diffuse LUMO (panel (b) in the figure). This state is indeed ready to partially accept electrons, and we can already anticipate that this will have consequences on the reaction mechanism. Before proceeding, however, we wish to remark that solvent exchange processes, which are likely to be related to the present case, can be divided into three stoichiometric mechanisms in which a change in the coordination number of the metal center is the order parameter. The increase, decrease, and kinetically undetectable behavior in the coordination number is termed to be associative, dissociative, and interchange modes, respectively. ${ }^{66}$ The dissociative mechanism proceeds with a low probability due to the long time scale of the mean lifetime of the associated moieties, e.g. water molecules, around the metal center. In the process, the kinetics of octahedral water coordination around the metal center triggers the interchange mechanism. Furthermore, the interchange mode can still be classified into two groups: associative $\left(\mathrm{I}_{a}\right)$ and dissociative $\left(\mathrm{I}_{d}\right)$ modes, according to whether or not the ligands entering or leaving the coordination shell trigger an exchange process between the first and the second coordination shell of the metal center. $^{67}$

The simulation protocol used here is identical to the ones previously discussed and will not be repeated. By constraining one of the two ligands to depart from the metal center, BME simulations give the free energy profile shown in Figure 8 .

A first noticeable feature is the fact that this reaction occurs with a significantly low free energy barrier $(\Delta F=5.65 \mathrm{kcal} / \mathrm{mol}$ $(0.25 \mathrm{eV}))$ with respect to all former simulations. By inspecting

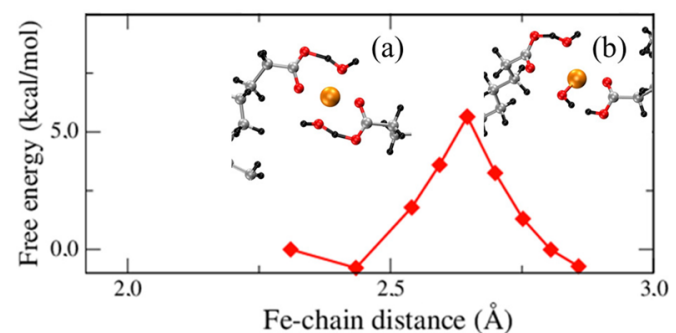

Figure 8. Free energy profile for the desorption of the chain in a Febased stearate in the presence of two water molecules. Inset (a) shows the transition state configuration with $\mathrm{H}_{2} \mathrm{O}$ molecules sharing a proton with the $-\mathrm{COO}^{-}$moieties of the ligands. Inset (b) shows the final $\mathrm{Fe}-\mathrm{OH}$ product. the trajectory, it is clear that the presence of water molecules is responsible for this. In fact, while the BME simulation proceeds, the completeness of the hydration shell of the metal center, initially in a $\mathrm{Fe}$ (III) configuration, gradually evolves to a planar configuration shown in the inset (a) of Figure 8. When this occurs, the water molecules turn out to be fully inserted between the two ligands of the stearate, forming strong hydrogen bonds with one of the two $\mathrm{O}$ atoms of the $-\mathrm{COO}^{-}$groups. The $\mathrm{Fe}$ cation, in these conditions, is coordinated to just one of the two oxygens of each $-\mathrm{COO}^{-}$ moieties, whereas a second $\mathrm{O}$ coordination is provided by the $\mathrm{H}_{2} \mathrm{O}$ molecule linking the metal center to the ligand. More precisely, at a $\mathrm{Fe}-\mathrm{COO}$ constrained distance of $2.65 \AA$, the square planar symmetry made by the oxygens of the carboxylate moieties reverts to a rhombus by shifting to the two opposite sides. The octahedral symmetry established by the water molecules at the beginning of the simulation is then disrupted, and the iron atom acquires a 4-fold coordination as specified above. This mechanism is what is termed interchange mode. ${ }^{66}$

At the transition state, the strong hydrogen bonds between the ligand and the $\mathrm{H}_{2} \mathrm{O}$ molecule give rise to a Zundel-like complex, in which a proton jumps continuously from the water molecule to the $-\mathrm{COO}^{-}$group. ${ }^{59-61}$ In this respect, water takes the role of a catalyst, facilitating the departure of the ligand of the stearate via the dissociation of $\mathrm{H}_{2} \mathrm{O}$ into a proton $\mathrm{H}^{+}$and a hydroxyl anion $\mathrm{OH}^{-}$. The proton binds to the $-\mathrm{COO}^{-}$group, which becomes a $-\mathrm{COOH}$, and eventually departs along with the ligand. Instead, the $\mathrm{OH}^{-}$binds to the metal center forming a $\mathrm{FeOH} \mathrm{NP}$ seed similar to the one found in former simulations, yet at variance with all previous cases, here the oxidation of the metal center is ensured by the (dissociated) water molecule and not by the $\mathrm{O}$ atoms belonging to the ligand. The depicted mechanism shows rather clearly that the retract of the hydrocarbon chain from the Fe center provokes a dissociative interchange mechanism $\mathrm{I}_{d}$, in agreement with the results reported in Table 1 of ref 64 . The sharp peak in the free energy profile coincides with the formation of these Zundel-like structures giving origin to the new chemical bonds. These, in turn, induce evident modifications of the electronic structure around the metal center. A comparison of the HOMO and LUMO states of the two main stages of the process, i.e. before and after the breakdown of the octahedral symmetry and the rearrangement of the water molecules, is given in Figure 9. Before the configurational change, the HOMO displays a mixing character due to the $d_{z}^{2}$ orbital of the Fe center and $1 b_{1}$ states typical of $\mathrm{H}_{2} \mathrm{O}$ molecules, plus minor contributions from the ligand. While the HOMO retains its $\mathrm{d}_{z}^{2}$ symmetry after the planar insertion of the $\mathrm{H}_{2} \mathrm{O}$ molecule between the metal and the ligand, the LUMO transforms into a $\mathrm{d}_{x y}$ orbital plus additional contributions on the $\mathrm{O}$ atoms of the coordinated water molecules. These states are characterized by corresponding eigenvalues located, on the energy scale of our DFT framework, at $-3.75 \mathrm{eV}$ (HOMO) and $-2.51 \mathrm{eV}$ (LUMO). Hence, the system has a gap of $1.23 \mathrm{eV}$, identical to the one of the octahedral configuration. Nonetheless, the planar configuration becomes more stable as the BME simulation proceeds beyond the $\Delta F=5.65 \mathrm{kcal} / \mathrm{mol}$ barrier, and the $\mathrm{HOMO}$ and LUMO eigenvalues initially located at -2.44 and $-1.21 \mathrm{eV}$, respectively, shift to the lower values reported above. This is accompanied by a qualitative change in the nature of the LUMO states from the typical LUMO of $\mathrm{H}_{2} \mathrm{O}$ to the $\mathrm{d}_{x y}$ orbital of Fe. 

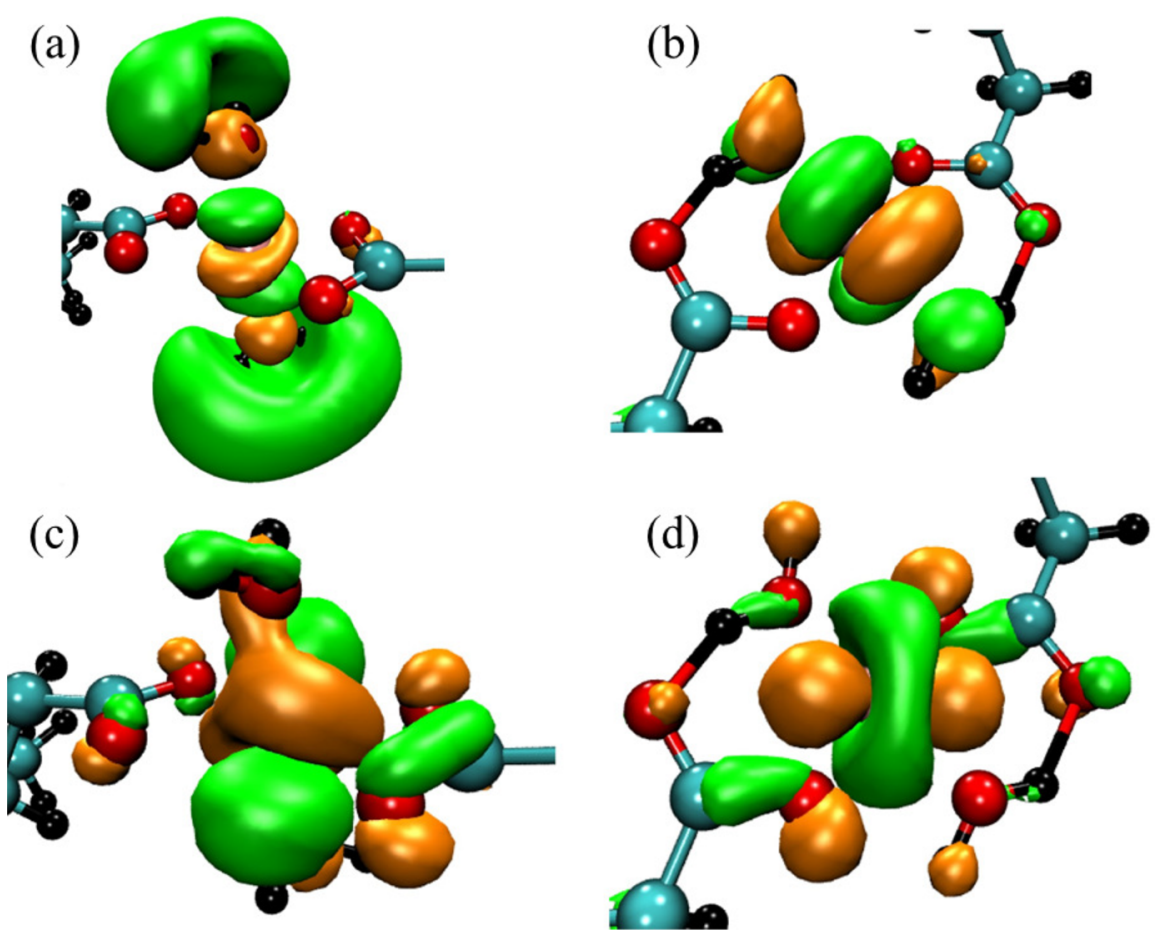

Figure 9. LUMO (top row) and HOMO (bottom row) orbitals of the Fe-based stearate in the presence of two water molecules in the octahedral $\mathrm{Fe}$ (III) configuration (panels a and c) and in the planar Fe(II) arrangement (panels b and d). Isosurfaces are shown at values of \pm 0.025 (e/ $\left.\AA^{3}\right)^{1 / 2}$ in orange (positive values) and green (negative values). The color code is identical to that of Figure 1 with carbon atoms in cyan.

In the case of the Co-based system, analogous BME simulations were started from an equilibrated system in which the Co atom is in an octahedral configuration similar to the one of $\mathrm{Fe}$ and sketched in Figure 1(c). The free energy profile obtained in this case is reported in Figure 10. A first

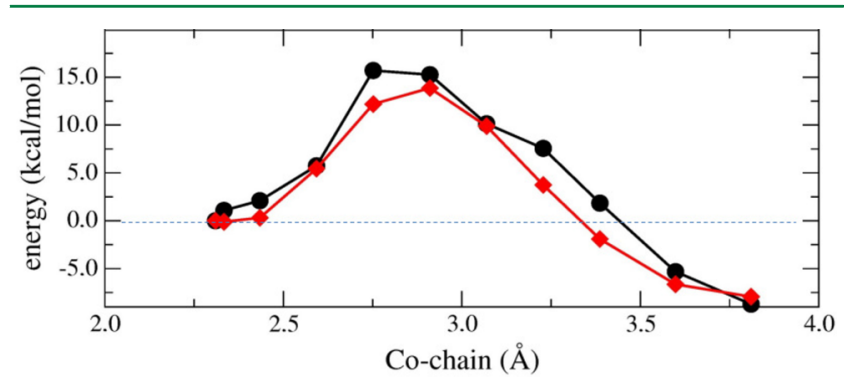

Figure 10. Free (red) and total (black) energy profiles for the desorption of the ligand in a fully hydrated Co-based stearate as a function of the reaction coordinate $d_{\mathrm{Co}-\mathrm{COO}}$.

noticeable feature is the presence of a free energy barrier of $\Delta F$ $=13.90 \mathrm{kcal} / \mathrm{mol}((0.60 \mathrm{eV})$ roughly twice larger than the case of the Fe-based stearate, occurring for a value of the Co-COO reaction coordinate of $2.91 \AA$. Also in this case, as opposed to the dry conditions, both the free (red curve in Figure 10) and the total (black curve in Figure 10) energy profiles show a decrease to zero after the transition state at $d_{\mathrm{Co}-\mathrm{COO}}=2.91 \AA$. The dissociation energy is then significantly decreased, and also in this case, the reason is the catalytic action of the water molecules in proximity of the metal center. Furthermore, the energy profile shows the exothermic nature of this particular reaction with an energy gain of $7.93 \mathrm{kcal} / \mathrm{mol}(0.34 \mathrm{eV})$ of the product with respect to the reactant, whereas its Fe-based analogue shows a minimal energy gain amounting to only 0.72 $\mathrm{kcal} / \mathrm{mol}(0.03 \mathrm{eV})$.

The reaction mechanism is similar to the one found for the Fe-based hydrated system, and its main stages are shown in Figure 11. The ligand desorption application proceeds in a way similar to the Fe-based system, with the $\mathrm{H}_{2} \mathrm{O}$ molecule leaving the octahedral configuration and inserting between the fatty acid chain and the metal center. Also in this case, the dissociation of a water molecule during the BME simulation leads to the protonation of the carboxylate group of the ligand after the formation of a Zundel-like complex. The detached proton eventually forms a stable bond with one of the $\mathrm{O}$ atoms of the fatty acid chain changing the terminal group from $-\mathrm{COO}^{-}$to $-\mathrm{COOH}$. As in the Fe case, the hydroxyl anion $\mathrm{OH}^{-}$binds to the Co center causing the hydroxylation and the formation of a $\mathrm{CoOH} \mathrm{NP}$ seed. In a way analogous to the $\mathrm{Fe}$ based stearate, the oxidation of the Co center is triggered by water which acts as a catalyst and contributes with its dissociation to a smoother departure of the ligand, yet the presence of two water molecules in the hydration shell of the $\mathrm{Co}$ atom inhibits the formation of pure $\mathrm{CoO}$ seeds with later consequences on their aggregation to form a NP.

Following the experimental suggestions, ${ }^{17,18,24}$ and in view of the larger displacements of the ligands observed in the case of the Co-based stearates in comparison with the Fe-based counterparts, we repeated this same simulation by changing the pre-equilibration procedure and time. An initial geometry relaxation within a preconditioned conjugate gradient (PCG) procedure was done to optimize the system with the two $\mathrm{H}_{2} \mathrm{O}$ molecules in the octahedral position of Figure 1(c) to get rid of any residual forces. This optimization step is the major difference in the preparation of the system with respect to a damped dynamics approach done, as opposed to the smooth damped dynamics used to relax the system in the former 


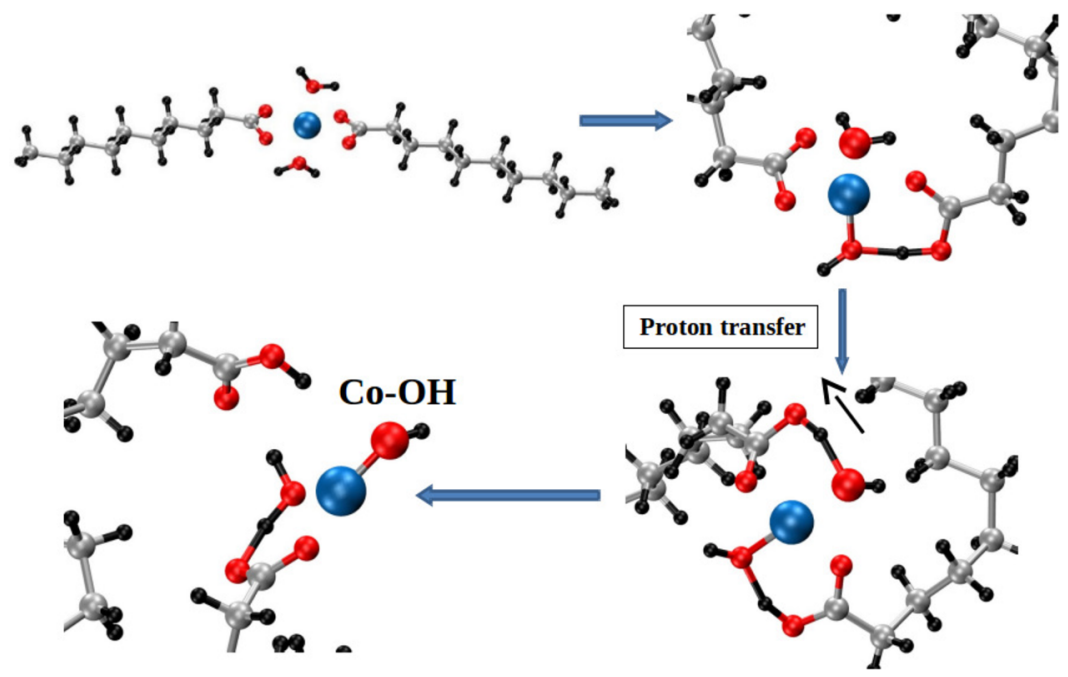

Figure 11. Main steps of the reaction pathway for a fully hydrated Co-based stearate. A protonation of the carboxylate group of the fatty acid chain occurs along with the formation of a $\mathrm{Co}-\mathrm{OH}$ moiety.

simulation. Then, dynamical simulations in the canonical NVT ensemble were performed at room temperature, thus heating instantaneously the system from 0 to $300 \mathrm{~K}$. This different treatment results in entirely different behavior and an alternative (complicated) reaction pathway. This suggests that the effects of the thermodynamical environment, and not only the level of hydration, are non-negligible on the dissociation process of Co-based stearates. Figure 12 summarizes the main steps of this dissociation mechanism.

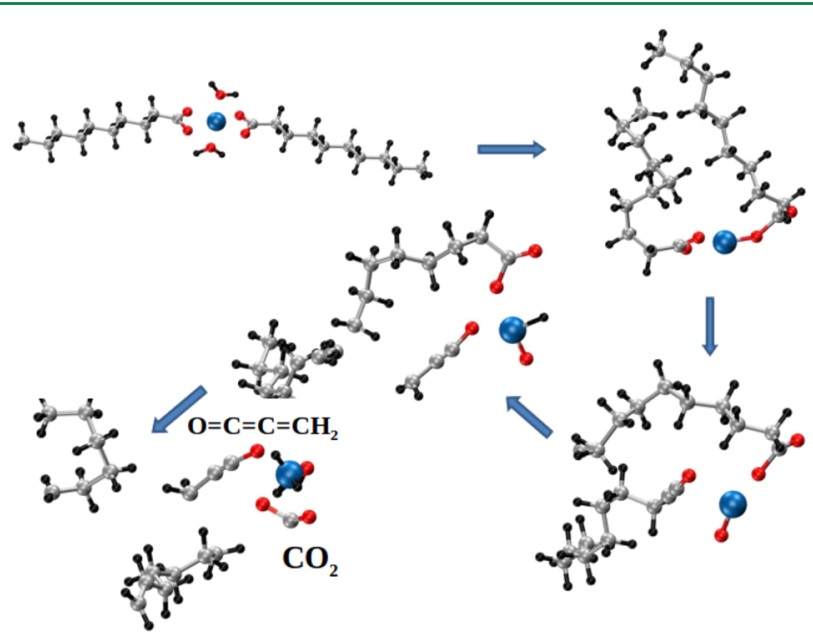

Figure 12. Second reaction mechanism found for the Co-based stearate in the presence of two water molecules. The desorption process terminates with the formation of a fragmented polycarbon chain, a $\mathrm{CO}_{2}$ molecule, and a protonated cobalt oxide moiety.

The major difference with respect to the previous result is the fragmentation of the fatty acid chain during the reaction that largely destabilizes the system. This process can occur with a negligible reaction barrier, as BME simulations have confirmed, and the overall reaction is exergonic, with a net energy gain of more than $20 \mathrm{kcal} / \mathrm{mol}$. Statistical fluctuations and conformational changes of the hydrocarbon chain are sufficient to disrupt the system and even to push away the added water molecules from the coordination shell of the Co center. The formation of $\mathrm{CoO}$ seed is not entirely jeopardized, as shown in Figure 12, but the ligand is irreversibly fragmented and can induce protonation of the $\mathrm{CoO}$ seed in an uncontrolled way, as well as the formation of $\mathrm{CO}_{2}$ molecules as byproducts. These hydrogen-carrying $\mathrm{CoO}$ seeds become, in turn, difficult to assemble in a metal oxide NP. This competing reaction channel can be interpreted as one of the main reasons for the experimental problems in the synthesis of Co-based NPs, along with related problems in size, shape, and morphology control, despite environmental and thermodynamical identical conditions used for the synthesis of Fe-based NPs. ${ }^{17,18,24}$

\section{CONCLUSION}

Our extensive computational studies have targeted the evaluation of the effect of the presence of water traces in the precursors stearate compounds used for the synthesis of metal oxide NPs. Our atomic-level insight has shown that the decomposition of $\mathrm{Fe}$ - and Co-stearates leads to the formation of metal oxide building blocks for NPs assembly. Such a reaction proceeds with a significantly lower activation barrier in the case of the presence of $\mathrm{H}_{2} \mathrm{O}$ molecules in the hydration shell of the metal center, in comparison with dry conditions or partial hydration. Both the coordination geometry around the metal center and the chemical nature of the metal (Fe or Co) play crucial roles in the mechanism and in the energetics of the dissociation reaction. In both dry and $1-\mathrm{H}_{2} \mathrm{O}$ conditions, $\mathrm{Fe}-\mathrm{O}$ seeds, precursors of the NP, can be generated for iron-based stearates, the major differences being the associated activation barrier and the catalytic role of water if present. Conversely, in the case of cobalt, even if water still plays a catalytic role, the free energy barrier for the dissociation is still more than twice larger than for $\mathrm{Fe}$-stearates in analogous conditions. Furthermore, fragmentation of the fatty acid chains can lead to the formation of $\mathrm{Co}-\mathrm{OH}$ moieties which can accept protons (or $\mathrm{H}$ atoms) from the disrupted ligand. These Co-based seeds, in turn, make the aggregation of the resulting NPs more difficult since they are much less controllable and affect shape, morphology, and the resulting properties of the synthesized NP. The assembly of $\mathrm{Co}$ (II)-hydroxides is in fact more problematic than the aggregation of the (nonprotonated) $\mathrm{Fe}$ (II,III)-oxides. We suggest that hydration has to be avoided and precursors carefully dried if Co-stearates have to be used for the NP synthesis. On the other hand, Fe-based stearates are 
less sensitive to the hydration conditions (humidity level of the experimental apparatus), and their hydration can even favor the formation of metal oxide building blocks. This is due to the catalytic action of water which, far from being an inert ingredient, plays an active role and does not jeopardize the synthesis process.

\section{ASSOCIATED CONTENT}

\section{S Supporting Information}

The Supporting Information is available free of charge on the ACS Publications website at DOI: 10.1021 /acs.jctc.7b00869.

Hybrid functional calculations comparative studies (PDF)

\section{AUTHOR INFORMATION}

\section{Corresponding Author}

*E-mail: mauro.boero@ipcms.unistra.fr.

\section{ORCID $\odot$}

Assil Bouzid: 0000-0002-9363-7240

Mauro Boero: 0000-0002-5052-2849

\section{Present Addresses}

${ }^{\ddagger}$ Department of Materials Science and Engineering, Izmir Institute of Technology, 35430 Urla, Izmir, Turkey.

"Chaire de Simulation à l'Echelle Atomique (CSEA), Ecole Polytechnique Fédérale de Lausanne (EPFL), CH-1015 Lausanne, Switzerland.

\section{Funding}

M.B. thanks Pôle HPC and Equipex Equip@Meso at the University of Strasbourg, Grand Equipement National de Calcul Intensif (GENCI) under allocation DARI-A2 A0020906092, and the LaBex "Nanoparticles Interacting with their Environment" (NIE) ANR-11-LABX-0058 NIE within the Investissement d'Avenir ANR-10-IDEX-0002-02. G.O. acknowledges financial support from Initiative d'excellence IdEX "Attractivite" of the University of Strasbourg.

Notes

The authors declare no competing financial interest.

\section{ACKNOWLEDGMENTS}

We are grateful to S. Bégin-Colin and B. P. Pichon for insightful discussions.

\section{REFERENCES}

(1) Gupta, A. K.; Gupta, M. Synthesis and Surface Engineering of Iron Oxide Nanoparticles for Biomedical Applications. Biomaterials 2005, 26, 3995-4021.

(2) Pamme, N.; Wilhelm, C. Continuous Sorting of Magnetic Cells via On-Chip Free-Flow Magnetophoresis. Lab Chip 2006, 6, 974-980.

(3) Qiao, R.; Yang, C.; Gao, M. Superparamagnetic Iron Oxide Nanoparticles: From Preparations to in vivo MRI Applications. J. Mater. Chem. 2009, 19, 6274-6293.

(4) Sun, S.; Zeng, H. Size-Controlled Synthesis of Magnetite Nanoparticles. J. Am. Chem. Soc. 2002, 124, 8204-8205.

(5) Frolov, G. I. Film Carriers for Super-High-Density Magnetic Storage. Tech. Phys. 2001, 46, 1537-1544.

(6) Kinge, S.; Crego-Calama, M.; Reinhoudt, D. N. Self-Assembling Nanoparticles at Surfaces and Interfaces. ChemPhysChem 2008, 9, 2042 .

(7) Kishore, P. N. R.; Jeevanandam, P. A Novel Thermal Decomposition Approach for the Synthesis of Silica-Iron Oxide Core-Shell Nanoparticles. J. Alloys Compd. 2012, 522, 51-62.

(8) Dinh, C. T.; Nguyen, T. D.; Kleitz, F.; Do, T. O. ShapeControlled Synthesis of Metal Oxide Nanocrystals. In Controlled
Nanofabrication: Advances and Applications; Pat Stanford Pubs.: Danvers, MA, 2012; pp 3278-367, DOI: 10.1201/b13155-11.

(9) Xia, Y.; Xiong, Y.; Lim, B.; Skrabalak, S. Shape-Controlled Synthesis of Metal Nanocrystals: Simple Chemistry Meets Complex Physics? Angew. Chem., Int. Ed. 2009, 48, 60-103.

(10) Gonzalez-Moragas, L.; Yu, S.-M.; Murillo-Cremaes, N.; Laromaine, A.; Roig, A. Scale-Up Synthesis of Iron Oxide Nanoparticles by Microwave-Assisted Thermal Decomposition. Chem. Eng. J. 2015, 281, 87-95.

(11) Dou, Q.; Ng, K. M. Synthesis of Various Metal Stearates and the Corresponding Monodisperse Metal Oxide Nanoparticles. Powder Technol. 2016, 301, 949-958.

(12) Üstünel, H.; Erkoc, S. Structural Properties and Stability of Nanoclusters. J. Comput. Theor. Nanosci. 2007, 4, 928-956.

(13) Xie, L.; Harris, D. K.; Bawendi, M. G.; Jensen, K. F. The Effect of Trace Water on the Growth of Indium Phosphide Quantum Dots. Chem. Mater. 2015, 27, 5058-5063.

(14) Franke, D.; Harris, D. K.; Xie, L.; Jensen, K. F.; Bawendi, M. G. The Unexpected Influence of Precursor Conversion Rate in the Synthesis of III-V Quantum Dots. Angew. Chem., Int. Ed. 2015, 54, 14299-14303.

(15) Franke, D.; Harris, D. K.; Chen, O.; Bruns, O. T.; Carr, J. A.; Wilson, M. W. B.; Bawendi, M. G. Continuous Injection Synthesis of Indium Arsenide Quantum Dots Emissive in the Short-Wavelength Infrared. Nat. Commun. 2016, 7, 12749-12749.

(16) Morones, J. R.; Elechiguerra, J. L.; Camacho, A.; Holt, K.; Kouri, J. B. The Bactericidal Effect of Silver Nanoparticles. Nanotechnology 2005, 16, 2346-2353.

(17) Demortiere, A.; Panissod, P.; Pichon, B. P.; Pourroy, G.; Guillon, D.; Donnio, B.; Bégin-Colin, S. Size-Dependent Properties of Magnetic Iron Oxide Nanocrystals. Nanoscale 2011, 3, 225-232.

(18) Gerber, O.; Pichon, B. P.; Ihiawakrim, D.; Florea, I.; Moldovan, S.; Ersen, O.; Begin, D.; Grèneche, J.-M.; Lemonnier, S.; Barraud, E.; Bégin-Colin, S. Synthesis Engineering of Iron Oxide Raspberry-Shaped Nanostructures. Nanoscale 2017, 9, 305-313.

(19) Zhang, H.-T.; Chen, X.-H. Controlled Synthesis and Anomalous Magnetic Properties of Relatively Monodisperse CoO Nanocrystals. Nanotechnology 2005, 16, 2288-2294.

(20) Lu, A.-H.; Salabas, E. L.; Schuth, F. Magnetic Nanoparticles: Synthesis, Protection, Functionalization, and Application. Angew. Chem., Int. Ed. 2007, 46, 1222-1244.

(21) Jana, N. R.; Chen, Y.; Peng, X. Size- and Shape-Controlled Magnetic (Cr, $\mathrm{Mn}, \mathrm{Fe}, \mathrm{Co}, \mathrm{Ni}$ ) Oxide Nanocrystals via a Simple and General Approach. Chem. Mater. 2004, 16, 3931-3935.

(22) Abrahamson, H. B.; Lukaski, H. C. Synthesis and Characterization of Iron Sterate Compounds. J. Inorg. Biochem. 1994, 54, 115130

(23) Pinna, N.; Niederberger, M. Surfactant-Free Nonaqueous Synthesis of Metal Oxide Nanostructures. Angew. Chem., Int. Ed. 2008, 47, 5292-5304.

(24) Baaziz, W.; Pichon, B. P.; Liu, Y.; Grene, J.-M.; Ulhaq-Bouillet, C. Tuning of Synthesis Conditions by Thermal Decomposition toward Core-Shell $\mathrm{Co}(\mathrm{x}) \mathrm{Fe}(1-\mathrm{x}) \mathrm{O} @ \mathrm{Co}(\mathrm{y}) \mathrm{Fe}(3-\mathrm{y}) \mathrm{O} 4$ and $\mathrm{CoFe} 2 \mathrm{O} 4$ Nanoparticles with Spherical and Cubic Shapes. Chem. Mater. 2014, 26, 5063-5073.

(25) Powell, H. K. J.; Nancollas, H. Coordination of Oxygen by Cobalt (II) Complexes in Aqueous Solution. A Calorimetric Study. J. Am. Chem. Soc. 1972, 94, 2664-2668.

(26) Wagner, B.; Reinen, D.; Brunold, Th. C.; Gudel, H. U. Iron(VI) in Tetrahedral Oxo Coordination: A Single Crystal EPR Study. Inorg. Chem. 1995, 34, 1934-1942.

(27) Ohashi, K.; Sasaki, J.; Yamamoto, G.; Judai, K.; Nishi, N.; Sekiya, H. Temperature Effecys on Prevalent Structures of Hydrated $\mathrm{Fe}^{+}$ Complexes:Infrared Spectroscopy and DFT Calculations of $\mathrm{Fe}^{+}\left(\mathrm{H}_{2} \mathrm{O}\right)_{n}(n=3-8)$. J. Chem. Phys. 2014, 141, 214307.

(28) Ikeda, T.; Boero, M.; Terakura, K. Hydration Properties of Magnesium and Calcium Ions from Constrained First Principles Molecular Dynamics. J. Chem. Phys. 2007, 127, 074503. 
(29) Ikeda, T.; Boero, M. Role of van der Waals Corrections in First Principles Simulations of Alkali Metal Ions in Aqueous Solution. J. Chem. Phys. 2015, 143, 194510.

(30) Palchoudhury, S.; An, W.; Xu, Y.; Qin, Y.; Zhang, Z.; Chopra, N.; Holler, R. A.; Turner, C. H.; Bao, Y. Synthesis and Growth Mechanism of Iron Oxide Nanowhiskers. Nano Lett. 2011, 11, 11411146.

(31) Xie, L.; Zhao, Q.; Jensen, K. F.; Kulik, H. J. Direct Observation of Early-Stage Quantum Dot Growth Mechanisms with HighTemperature Ab Initio Molecular Dynamics. J. Phys. Chem. C 2016, 120, 2472-2483.

(32) Baaziz, W.; Pichon, B. P.; Fleutot, S.; Liu, Y.; Lefevre, C.; Greneche, J.-M.; Toumi, M.; Mhiri, T.; Begin-Colin, S. Magnetic Iron Oxide Nanoparticles: Reproducible Tuning of the Size and NanosizedDependent Composition, Defects, and Spin Canting. J. Phys. Chem. C 2014, 118, 3795-3810.

(33) Car, R.; Parrinello, M. Unified Approach for Molecular Dynamics and Density-Functional Theory. Phys. Rev. Lett. 1985, 55, 2471-2474.

(34) Kohn, W.; Sham, L. J. Self-Consistent Equations Including Exchange and Correlation Effects. Phys. Rev. 1965, 140, A1133A1138.

(35) CPMD; Copyright IBM Corp. 1990-2016, Copyright MPI für Festkörperforschung Stuttgart 1997-2001. http://www.cpmd.org/ (accessed Dec 14, 2017).

(36) Becke, A. D. Density-Functional Exchange-Energy Approximation with Correct Asymptotic Behavior. Phys. Rev. A: At., Mol., Opt. Phys. 1988, 38, 3098-3100.

(37) Lee, C.; Yang, W.; Parr, R. G. Development of the Colle-Salvetti Correlation-Energy Formula into a Functional of the Electron Density. Phys. Rev. B: Condens. Matter Mater. Phys. 1988, 37, 785-789.

(38) Troullier, N.; Martins, J. L. Efficient Pseudopotentials for PlaneWave Calculations. Phys. Rev. B: Condens. Matter Mater. Phys. 1991, 43, 1993-2006.

(39) Goedecker, S.; Teter, M.; Hutter, J. Separable Dual-SpaceGaussian Pseudopotentials. Phys. Rev. B: Condens. Matter Mater. Phys. 1996, 54, 1703-1710.

(40) Hartwigsen, C.; Goedecker, S.; Hutter, J. Relativistic Separable Dual-Space-Gaussian Pseudopotentials from H to Rn. Phys. Rev. B: Condens. Matter Mater. Phys. 1998, 58, 3641-3662.

(41) Krack, M. Pseudopotentials for $\mathrm{H}$ to Kr Optimized for GradientCorrected Exchange-Correlation Functionals. Theor. Chem. Acc. 2005, 114, 145-152.

(42) Barnett, R. N.; Landman, U. Born-Oppenheimer MolecularDynamics Simulations of Finite Systems:Structure and Dynamics of $\left(\mathrm{H}_{2} \mathrm{O}\right)_{2}$. Phys. Rev. B: Condens. Matter Mater. Phys. 1993, 48, 20812097.

(43) Grimme, S. Semiempirical GGA-type Density Functional Constructed with a Long-range Dispersion Correction. J. Comput. Chem. 2006, 27, 1787-1799.

(44) Sprik, M.; Ciccotti, G. Free Energy from Constrained Molecular Dynamics. J. Chem. Phys. 1998, 109, 7737-7744.

(45) Ciccotti, G.; Kapral, R.; Vanden-Eijnden, E. Blue Moon Sampling, Vectorial Reaction Coordinates, and Unbiased Constrained Dynamics. ChemPhysChem 2005, 6, 1809-1814.

(46) Perdew, J. P.; Ernzerhof, M.; Burke, K. Rationale for Mixing Exact Exchange with Density Functional Approximations. J. Chem. Phys. 1996, 105, 9982-9985.

(47) Steinmetz, M.; Grimme, S. Benchmark Study of the Performance of Density Functional Theory for Bond Activations with (Ni,Pd)Based Transition-Metal Catalysys. ChemistryOpen 2013, 2, 115-124.

(48) Özdamar, B.; Massobrio, C.; Boero, M. Stability and Destabilization Processes in the Formation of Ferrocene-based Metal-Organic Molecule-Metal Nano-Junctions. J. Phys. Chem. C 2016, 120, 13825-13830.

(49) Johnson, B. G.; Gill, P. M. W.; Pople, J. A. The Performance of a Family of Density Functional Methods. J. Chem. Phys. 1993, 98, 56125626.
(50) Probert, M. I. J. Improved Algorithm for Geometry Optimisation Using Damped Molecular Dynamics. J. Comput. Phys. 2003, 191, 130-146.

(51) Nosé, S. A Molecular Dynamics Method for Simulations in the Canonical Ensemble. Mol. Phys. 1984, 52, 255-268.

(52) Nosé, S. A Unified Formulation of the Constant Temperature Molecular Dynamics Methods. J. Chem. Phys. 1984, 81, 511-519.

(53) Hoover, W. G. Canonical Dynamics: Equilibrium Phase-space Distributions. Phys. Rev. A: At., Mol., Opt. Phys. 1985, 31, 1695-1697.

(54) Martyna, G. J.; Klein, M. L.; Tuckerman, M. Nosé-Hoover Cains: The Canonical Ensemble via Continuous Dynamics. J. Chem. Phys. 1992, 97, 2635-2643.

(55) Pokrovski, G. S.; Schott, J.; Farges, F.; Hazemann, J.-L. Iron (III)-silica interactions in aqueous solution: Insights from X-ray absorption fine structure spectroscopy. Geochim. Cosmochim. Acta 2003, 67, 3559-3573.

(56) Bullock, R. M.; Samsel, E. G. Hydrogen Atom Transfer Reactions of Transition-Metal Hydrides. Kinetics and Mechanism of the Hydrogenation of Alpha-Cyclopropylstyrene by Metal Carbonyl Hydrides. J. Am. Chem. Soc. 1990, 112, 6886-6898.

(57) Boero, M.; Parrinello, M.; Terakura, K. Ziegler-Natta Heterogeneous Catalysis by First Principles Computer Experiments. Surf. Sci. 1999, 438, 1-8.

(58) Boero, M.; Parrinello, M.; Hüffer, S.; Weiss, H. First Principles Study of Propene Polymerization in Ziegler-Natta Heterogeneous Catalysis. J. Am. Chem. Soc. 2000, 122, 501-509.

(59) Zundel, G.; Metzger, H. Energiebänder der tunnelnden Überschuss-Protonen in flüssigen Säuren - eine IR-spektroskopische Untersuchung der Natur der Gruppierungen $\mathrm{H}_{5} \mathrm{O}^{2+}$. Z. Phys. Chem. $1968,58,225-245$.

(60) Marx, D.; Tuckerman, M. E.; Hutter, J.; Parrinello, M. The Nature of the Hydrated Excess Proton in Water. Nature 1999, 397, 601-604.

(61) Boero, M.; Ikeshoji, T.; Terakura, K. Density and Temperature Dependence of Proton Diffusion in Water: A First-Principles Molecular Dynamics Study. ChemPhysChem 2005, 6, 1775-1779.

(62) Henkelman, G.; Arnaldsson, A.; Jónsson, H. A Fast and Robust Algorithm for Bader Decomposition of Charge Density. Comput. Mater. Sci. 2006, 36, 354-360.

(63) Cantu, D. C.; McGrail, B. P.; Glezakou, V.-A. Formation Mechanism of the Secondary Building Unit in a Chromium Terephthalate Metal-Organic Framework. Chem. Mater. 2014, 26, 6401-6409.

(64) Helm, L.; Merbach, A. E. Water Exchange on Metal Ions: Experiments and Simulations. Coord. Chem. Rev. 1999, 187, 151-181.

(65) Houghton, R. P. Metal Complexes in Organic Chemistry; Cambridge University Press: Cambridge, 1979.

(66) Langford, C. H.; Gray, H. B. Ligand Substitution Processes; W. A. Benjamin Inc.: New York-Amsterdam, 1965.

(67) Swaddle, T. W. On the Mechanism of Octahedral Substitution. Comments Inorg. Chem. 1991, 12, 237-258. 